\title{
Encyclopedia of \\ Business Analytics and Optimization
}

John Wang

Montclair State University, USA

\author{
Volume IV \\ Op-So
}


Managing Director:

Production Editor:

Development Editor:

Acquisitions Editor:

Typesetter:

Cover Design:
Lindsay Johnston

Jennifer Yoder

Austin DeMarco

Kayla Wolfe

Christina Barkanic, Michael Brehm, John Crodian,

Lisandro Gonzalez, Christina Henning, Deanna Jo Zombro

Jason Mull

Published in the United States of America by

Business Science Reference (an imprint of IGI Global)

701 E. Chocolate Avenue

Hershey PA 17033

Tel: 717-533-8845

Fax: 717-533-8661

E-mail: cust@igi-global.com

Web site: http://www.igi-global.com

Copyright (C) 2014 by IGI Global. All rights reserved. No part of this publication may be reproduced, stored or distributed in any form or by any means, electronic or mechanical, including photocopying, without written permission from the publisher. Product or company names used in this set are for identification purposes only. Inclusion of the names of the products or companies does not indicate a claim of ownership by IGI Global of the trademark or registered trademark.

Library of Congress Cataloging-in-Publication Data

Encyclopedia of business analytics and optimization / John Wang, editor.

pages $\mathrm{cm}$

Includes bibliographical references and index.

Summary: "This reference confronts the challenges of information retrieval in the age of Big Data by exploring recent advances in the areas of knowledge management, data visualization, interdisciplinary communication, and others"-- Provided by publisher.

ISBN 978-1-4666-5202-6 (hardcover) -- ISBN 978-1-4666-5203-3 (ebook) -- ISBN 978-1-4666-5205-7 (print \& perpetual access) 1. Management--Mathematical models. 2. Decision making--Mathematical models. 3. Business planning--Mathematical models. 4. Big data. I. Wang, John, 1955-

HD30.25.E53 2014

658.4'038--dc23

2013046204

British Cataloguing in Publication Data

A Cataloguing in Publication record for this book is available from the British Library.

All work contributed to this book is new, previously-unpublished material. The views expressed in this book are those of the authors, but not necessarily of the publisher.

For electronic access to this publication, please contact: eresources@igi-global.com. 


\title{
Sentiment Analysis in Supply Chain Management
}

\author{
Lincoln C. Wood \\ Auckland University of Technology, New Zealand \& Curtin University, Australia
}

\author{
Torsten Reiners \\ Curtin University, Australia
}

\section{Hari S. Srivastava \\ PNG University of Technology, Papua New Guinea}

\section{INTRODUCTION}

The supply chain is a sequence of activities which are conducted in separate companies. Materials flow along the supply chain progressively becoming transformed into a product that a consumer wishes to buy. On the other hand, information is transferred from consumers to producers, impacting on the future delivery plans for the suppliers. Ideally, feedback about the behaviour of the end consumer should be driving the coordinated behaviour of the supply chain. When consumers begin to buy more products, the sensible initiative for the supply chain to do is to match the demand with an increase of the product output in a process of demand and supply integration to improve operational efficiency (Esperetal., 2010). However, a well-studied phenomenon known as the bullwhip effect can often be observed. This is where significant fluctuations occur in the output of members along the supply chain, as members further away from the consumers tend to overreact to changes in the final marketplace; even in response to just small changes in consumer demand (Lee et al., 1997). This is largely due to the supply chain members' lack of information about market-based activities. This phenomenon makes coordination and management of the supply chain challenging and creates additional costs and reduces chain responsiveness. Rather actual market-based information being shared along the supply chain, it is more common that suppliers take orders from consumers as an indication of market demand. As a result, many firms find themselves confronted with potential asymmetries of information along the supply chain and fail to respond efficiently on the market-based demand by responding to orders being placed by their direct consumers (O'Leary, 2011). This inability to determine consumer demand changes can be overcome using analysis of social media and opinions posted online.

Analysis of social media can be used to sufficiently predict social behaviour (Abbasi et al., 2012). The use of 'sentiment analysis' or 'opinion mining' can allow firms to derive an understanding of changes in consumer demand or preferences as expressed in social media, . Thus, 'demand sensing' of market-based demand allows firms to detect shifts or changes in trends in market-based demand (rather than orders from consumers) which then can feed into planning processes without requiring cooperation or coordination with other firms in the supply chain (Qin, 2011). Using business analytics approach of textual 'sentiment analysis' creates the opportunity to ultimately enable the prediction of sales. At present, commercial tools have been more readily applied to marketing and sentiment analysis rather than focus on supply management applications (Zitnik, 2012).

Sentiment analysis has great value for all suppliers independent of their distant from the actual end consumers. Suppliers closer to consumers, such as those in food and beverage supply chains, 
tend to have well-established information-sharing connections that help supply chain members to be more aware of consumer demand changes and respond accordingly; this is exemplified in the Beer Game (Sterman, 1989). However, all suppliers can benefit from both forecasting and sentiment analysis as a large proportion of their supply is directed through their supply chain; a change in demand for one product or category can have significant ramifications and thus should be monitored. In some cases, firms directly serves consumers (such as supermarkets in food and beverage supply chains) may be reluctant to share insights about consumer demand; in these cases, sentiment analysis can prove to be exceedingly valuable to suppliers in short supply chains.

Suppliers that are distant from the consumer are disadvantaged by the relative difficulty in retrieving or accessing consumer demand information; sentiment analysis can prove to be advantageous in this respect. This value is, however, balanced by the fact that their output through any one supply chain going into one product/category that is affected by consumer shifts is a smaller proportion of their overall output. Thus, a shift in consumer demand for a particular product/category may have a smaller impact on their overall business, yet sentiment analysis may be the only practical method for them to gain insight and forewarning that this shift is about to occur in the first place.

In this chapter, we evaluate existing methods of gaining consumer demand information but focus on sentiment analysis as a key approach for firms without direct consumer contact, making the approach suitable for firms that are removed from consumers and marketplaces. We introduce supply chain management and other relevant concepts to provide context to the reader. We use the Beer Game (Sterman, 1989) as an example for a simple supply chain, allowing users to easily understand how information can be shared in supply chains, the overall effect of sharing information, and how delays in information affect the overall performance.

\section{BACKGROUND}

Supply chain management is the management of different companies and activities relating to the flow of materials and information in the supply chain. All material is harvested or extracted, processed, formed into components, which are then assembled into final goods, which undergo physical transportation and distribution to locations where consumers wish to buy them in the end marketplace. Supply chains have two important reverse flows from the consumer to the members of the supply chain. First in a reverse logistics process damaged or defective products flow from consumers for repair or disposal. Second, there is a flow of information about consumer demand, quantity requirements, and product features to manufacturers. The consumer demand is 'independent demand' as it cannot be directly influenced by the supply chain. Using the independent demand and the production plan and required components or products allow firms to calculate the 'dependent demand' for various components and products in the supply chain that are used in these finished goods (Helms, Ettkin, \& Chapman, 2000). The difficulty in coordinating and managing the whole supply chain lies in the delays in the transfer of information to suppliers and manufacturers, preventing provision of relevant and accurate information to aid decision making.

Consumer demand in the marketplace is not equivalent to orders transferred along the supply chain. Consumer demand can remain unfulfilled simply due to retailers failing to hold desired inventory; e.g., there may be consumer demand for blue and red coloured jackets yet consumers may be buying blue jackets only as there are no red jackets in stock. The blue jacket stocks are replenished and this provides an erroneous indication (when observing order information) that there is only consumer demand for blue jackets, leaving consumer demand for red jackets unperceived by retailers and, by extension, unperceived by the supply chain. 
Figure 1. The supply chain in the Beer Game, with delays in information transmission and product distribution

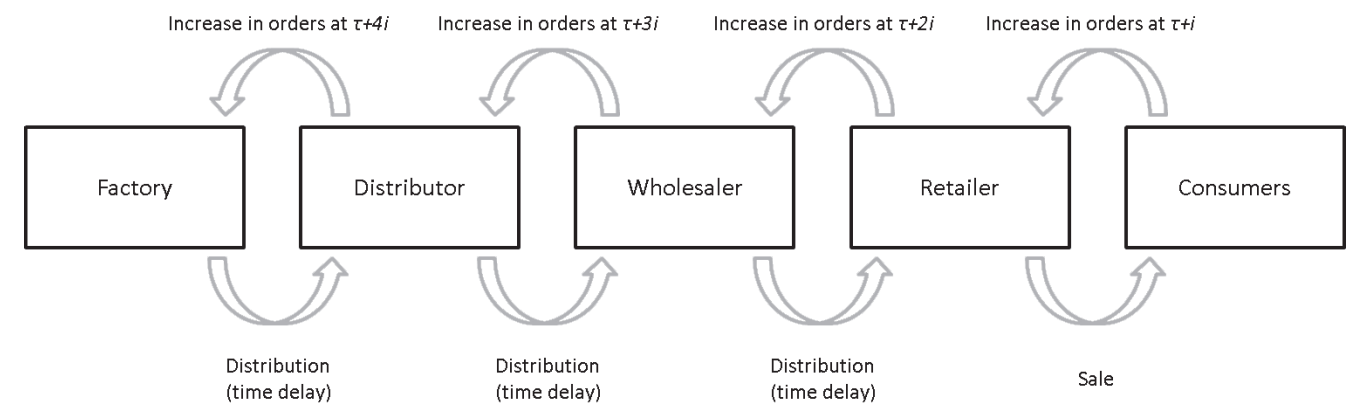

The Beer Game was developed to teach and demonstrate these dynamics within a simplified supply chain; constituting several companies and processes. The key structural elements of the game are the inventory held within each player facility, the delay and movement of materials to consumers, and a delay in transferring information from consumers through to each company (Figure 1). The instructor causes static consumer demand to increase once at time $\tau$ (albeit, by a significant amount), before becoming static; consumers react with larger orders at time $\tau+1$. The retailer observes this change yet is unable to communicate with the rest of the supply chain except through placement of orders. Note that when played in the face-to-face classroom with tokens, or counters, it is possible to see the actual amount of inventories that other people in the supply chain hold. Surprisingly, few students seem to make use of this information; a fact that is also given in real supply chains with an open sharing policy for information.

In the game, information about the change in demand is significantly delayed in the transmission over the supply chain while orders are being transferred down the supply chain from the retailer to the factory or manufacturer respectively. There is another delay as the materials flow through the supply chain from the factory to the retailer. As a result most of the players experience stock outs, backorders, and significant costs during the course of the simulation as they are unable to meet the demand of their consumers. The further the user is away from the consumer or retailer, the more significant the oscillations in the inventory levels and order sizes; i.e., the bullwhip effect is manifested (Figure 2). Eventually, players overreact and their situation changes from having no stock to possessing excessive levels of stock, throughout the supply chain.

There are many approaches for sharing information along the supply chain; including sharing forecasting data, inventory levels, sale trends, promotional events, or implementation of point of sale (POS) capturing technology like barcodes or radio frequency identification (RFID) tags in products. This means that every time a product is handed to a consumer, a sale record is kept in the information system. Traditionally, this information was used only by the retailer to support the placement of an order for goods from the supplier. Several methods have been employed to increase visibility, predominantly focusing on two tiers of the supply chain and more frequently used closer to the retail end of the supply chain. These methods include information sharing betweenERP systems, electronic data interchange (EDI), e-messaging systems, Just-in-time II (JIT II), vendor managed inventory (VMI), and collaborative planning forecasting and replenishment (CPFR).

- Increasingly, ERP systems can connect computer systems over the supply chain; i.e., including suppliers. In effect, such 
Figure 2. The pattern of increasing order sizes and the oscillation in the inventory levels which are more substantial the further the firm is from the consumers. This is the bullwhip effect

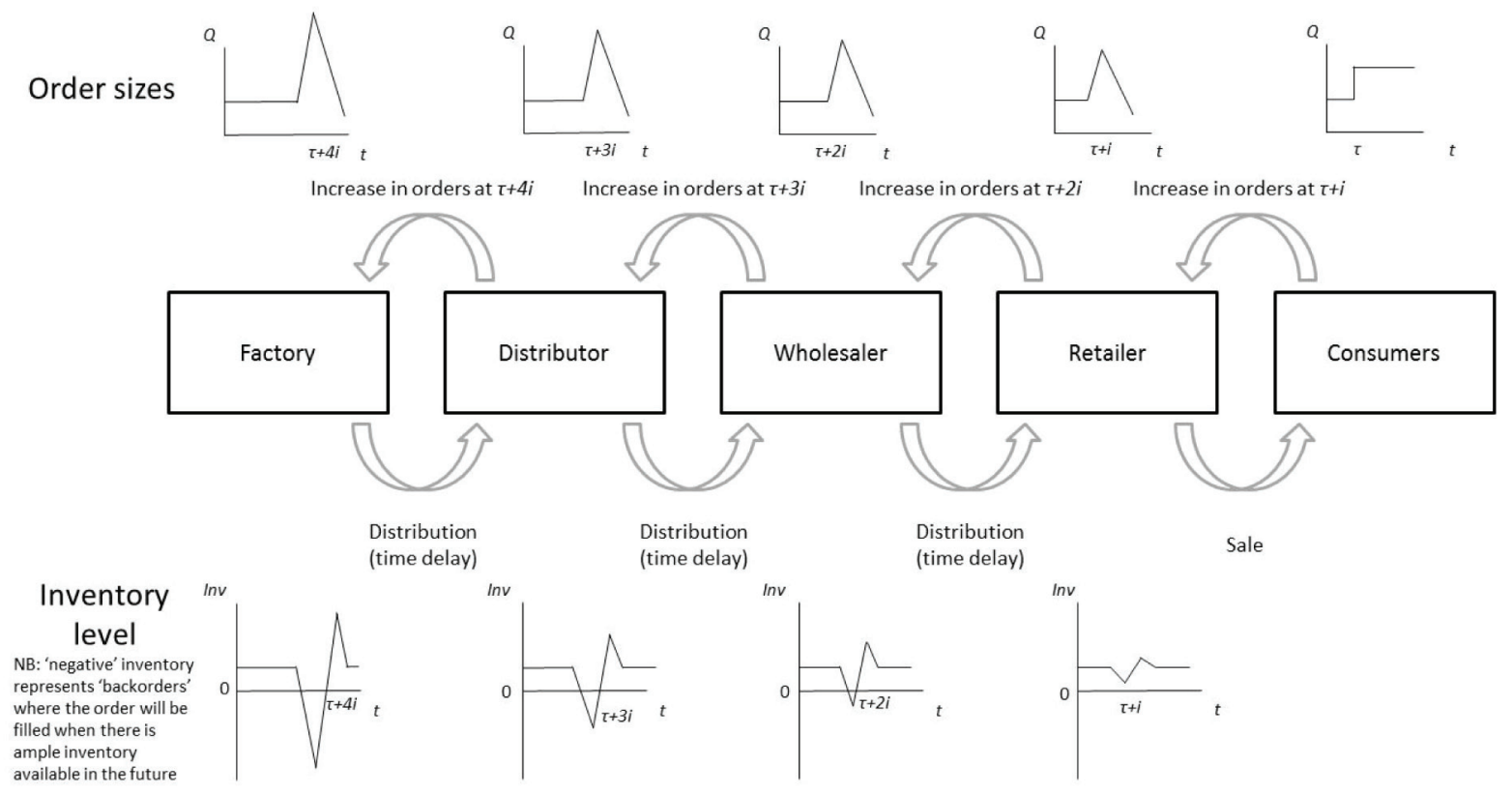

systems provide a direct window for the supplier to see through the fog of what is occurring at the retailer and begin to get an understanding of the actual POS data and the demand in the marketplace.

- $\quad$ Empowered suppliers in JIT II systems embed employees at consumer premises to directly place orders and manage the inventory at, or from, onsite. JIT II can incorporate VMI to enable suppliers to plan for the availability of products, reducing supply chain costs. The supplier is fully responsible for maintaining inventory according to negotiations with the buyer, leading to reduced inventory throughout the supply chain and improved service levels.

- Another methodology that has emerged over the last decade is CPFR, which enhances visibility between two tiers of the supply chain. It improves the forecast quality and the resulting planning decisions to fulfil the consumer demands. Planned purchase orders are jointly determined in advance, allowing supply managers more time to prepare for anticipated changes in consumer demand. Exceptions to the plan are analysed to understand their occurrence and how to implement appropriate reactions and improvements for the next time.

All of these approaches have the common theme of requiring collaboration with business partners along the supply chain, forcing a firm to rely on their partners. Retailers must be open to sharing POS data; trading partners must install EDI-compatible systems; consumers must accept your staff in JIT II systems or vendor involvement in VMI; CPFR by definition requires collaboration. When a firm is distant from consumers or is faced with uncooperative supply chain partners such techniques are difficult to implement.

Sentiment analysis allows a firm to overcome reliance on partners and collaboration with other firms. One of the key challenges for a firm is to achieve a deeper understanding of the consumers' behaviours, changes in the marketplace, and likely trends in the near future. The ability to analyse how consumers think or feel about particular products 
(or inventory non-availability, as demonstrated in the example of red jackets earlier) can be of advantage to the suppliers of components or materials used in those products; and goes beyond transferring information about sales. Without being able to understand or have visibility over the actual sales, firms have to understand how consumers think and feel about a certain product; an advantage to the suppliers of components or materials used in those products. (Note, however, that this requires an understanding of how the firm's output is incorporated into key products and supply chains; being a separate discussion beyond the scope of this chapter.)

Data mining is commonly used to analyse raw data to understand patterns and trends; e.g., sales data. With the rise of Web 2.0/3.0 as well as the Social Web, and significant increases in user-generated material (e.g., blogs, wikis, and discussion forums), a supply chain analyst can incorporate text mining to look for patterns in this massive body of text to extract useful information. However, unlike data mining information in text mining is less amenable to processing by algorithms.

\section{MAIN FOCUS OF THE ARTICLE}

Being able to understand what is being said about products and understanding consumer opinions about these products on the Internet can be important. Consumer opinions and reviews posted online can be used to predict sales and revenue indicating that changes in sentiment analysis can be used to predict changes in supply requirements over the supply chain (Qin, 2011; Yu \& Kak, 2012). Thus, aggregation of opinions can help business managers to identify, understand, and react to changes of attitudes over various dimensions such as time or geography; these summaries can also enable analysis of trends and patterns (Tsytsarau $\&$ Palpanas, 2012). Analysing the written words of people for sentiment analysis differs from analysis of statistical data, which is a far more common approach in supply chain management. Sentiment analysis requires a new set of capabilities and tools like natural language processing (Nadkarni et al., 2011), machine learning, supervised learning, or a hybrid of these methods (Prabowo \& Thelwall, 2009). While data mining numerically processes data to extract information and discover structures; sentiment analysis focuses on the meaning inferred by the order of words and context. Relevant steps in extracting information from text include, among others, the interpretation of words, tagging the part of speech, ignoring stop words, and separating out crucial and not-useful parts of the text. The result must be precise and comprehensible; thus transferred to the terminology used within the supply chain using lexical databases (WordNet) and thesauri for detecting synonyms. WordNet has several extensions supporting sentiment analysis; e.g., labels describing the polarity and objectivity (Baccianella et al., 2010) and labels representing different affective categories like emotion, cognitive state, or feeling (Strapparava \& Valitutti, 2004).

\section{Technologies}

There are four main technologies that help with concept identification in text: (1) Tagging 'Part of Speech' annotates sequences of text with the determined 'part' of the speech, e.g., noun or verb (Allen, 1985); (2) 'Entity Tracking' connects the same object identified differently throughout a text (e.g., USA for "United States of America"); (3) 'Named Entity Recognition' examines named entities (i.e., proper nouns) based on the entity lists being generated (Tjong Kim Sang \& De Meulder, 2003); and (4) 'Relation Extraction' examines the sentence structure and infers relationships between verbs and objects, and exploiting elements of the sentence structure (Koenig et al., 2008).

Analysis of concepts in natural language, assuming unstructured or semi-structured text, can take several forms. Formal concept analysis is a mathematical approach, using lattice theory and partially ordered sets to help determine the context 
using tuples (a set of entities, attributes, and the binary relationships between them) (Wille, 2009). Concept-based indexing uses a semantic network to determine crucial concepts based on semantic relatedness within the network (Boese et al., 2013). Latent semantic analysis assumes hidden coherence in text and uses analysis with correlation matrix and word occurrences (Landauer, 2007). Transformations are applied to return the most important terms and concepts in the text.

In addition to understanding meaning of words, sentiment analysis also requires understanding of the emotion or the meaning and subjectivity behind the written words (Montoyo et al., 2012). Thus, sentiment analysis allows encapsulation of positive or negative responses and changes (Madison et al., 2012) and understanding the strength of expression, following a supervised classification task (Taboada et al., 2011). Sentiment analysis has three constituent tasks: (1) subjectivity classification, (2) the sentiment classification, (3) and either opinion holder extraction or object/feature extraction, depending on the Analyst's requirements. The tasks can be undertaken at different levels of granularity in the analysis (Kumar \& Sebastian, 2012); fundamentally, therefore, analysing sentiment about a particular product (e.g., a particular brand and model of a laptop) is merely conducted at a different level of granularity than the analysis of a particular product feature (e.g., the inclusion of a digitizer or stylus with a tablet PC).

Subjectivity classification is the analysis of underlying meaning of phrases, separating those that are factual from those that are subjective, or opinionated. Clearly, this method is required to create a subset of text for further analysis. With such a piece of text it is necessary for it to undergo sentiment classification where it is classified as positive or negative sentiment. These can be either binary (e.g., positive/negative) or may be have multiple levels of strength (e.g., extremely negative, mostly negative, neutral, mostly positive, or extremely positive). This information can either be connected to a person or a feature. When opinion holder extraction is undertaken there is an effort to connect this information to a particular person. Object/feature extraction refers to connecting the sentiment information to a product or something about the project, which may help marketing or production (Kumar \& Sebastian 2012).

Previous work was included in the analysis of reviews trying to understand the meaning and the orientation of the sentiments of different analysed sentences. This can then be compared with the expert ratings and actual numeric user ratings and reviews; providing greater opinion analysis to support product design (Liu et al., 2013), and exemplary object/feature extraction.

The following examples demonstrate the value of comments and reviews to predict market changes; i.e., if aligned with other collected data like the number of clicks / agreements by other users or the site of publication. The examples also demonstrate the ambiguity of text and, therefore, the inadequacy of merely using data mining and key word recognition instead sentiment analysis.

1. "The quality of the lens in the new model of the camera is a clear setback." Comment approved of by $70 \%$ of 12,000 users.

2. "Great. I should not have bought the new model of $[\mathrm{X}]$ phone."

3. "I would recommend the product." Comment on a private Web-site.

4. "I suggest adding this product to your wish list for Christmas." Posted on the New York Times Website.

Comment 1 indicates that users disapprove the quality of the product over the previous model. This feedback is important for the supplier to improve the feature, but also to adjust the forecast as the number of sales may decrease; i.e., the large visibility and approval of negative comments may influence possible buyers to purchase another product. Comment 2 is difficult to analyse as the first part is positive (i.e., "Great"), while the second part is negative (i.e., "I should not have bought [...]"). This indicates a negative evaluation of the product that requires further investigation 
about reasons and actions to be taken. Comment 3 and 4 both recommend the product, while Comment 4 will likely cause an increase in sales due to the prominent placement of the review. The supplier should expect an increase in demand and therefore prepare for larger order quantities. The seasonal reference could imply that the sale numbers drop after Christmas; the market should be closely observed. This application of sentiment analysis is about determining likely periods of changed demand.

\section{Example}

Referring back to the Beer Game supply chain (Figure 1). At time $\tau$ when the shift in demand occurs, this will be reflected by an increase in user postings on Twitter, blogs, and product reviews. This increase in volume along with admiring comments creates a swell of positive sentiment. The manufacturing facility is able to determine that this is happening at the same time as the shift in consumer demand occurs. This can be accomplished independently, with no cooperation or coordination of information sharing with others in the supply chain. Thus, at time $\tau+i$ the manufacturer is capable of determining that there is an increase in consumer demand (while being unable to translate this into an exact value); faster than at time $\tau+3 i$ where the information would otherwise (in a perfectly functioning supply chain with no distortion of information caused by the bullwhip effect) reach the manufacturer. This can be accomplished by the Factory using specialised 'Demand Analysis' software (e.g., SAP's SCM module). After time $\tau$, when the Demand Analyst is able to perceive a noticeable increase in positive sentiment for the particular brand of beer (Figure 2), production for the beer can be increased (or, additional orders can be placed for raw materials), eliminating needless delays later. If a firm is manufacturing packaging for the Factory it is clear what the packaging will be used for and which type of products it is going to be used for in the marketplace. The packaging manufacturer will be able to use commercially-available sentiment analysis tools to gauge consumer- and marketbase responses. If, over time, increasingly strong, positive sentiment is being detected, the analyst is able to correlate this to increasing demand for the product, and, by dependent demand, for the focal firm's packaging.

Food and beverage supply chains (such as those exemplified in the Beer Game) tend to be short, providing manufacturers and producers with the ability to access market/demand data with relative ease, providing competitive advantage. The same principle can be applied in assembly firms. A firm that makes components used within products for a particular branded consumer electronics device can determine market sentiment and understand when their supply base needs to be expanded in anticipation of an increase in demand flowing through their supply chain in the future.

Furthermore, sentiment analysis is also able to provide indication of dangerous divergences between consumer demand and orders placed; when orders from consumer firms are increasing while the measureable sentiment about the end products decreases significantly, a manufacturer may be warned to reduce manufacturing or to plan for lower levels of inventory. An example is provided by Cisco's write-off of $2.25 \mathrm{~b}$ USD of inventory in 2001. Cisco's suppliers and contractors had "accumulated a large amount of inventory for months without factoring in the demand for Cisco's products" (Lee, 2004, p. 9). However, using sentiment analysis, the change in sentiment and resulting decrease in the marketplace could have been tracked and used as input into Sales and Operations Planning (S\&OP) decisions to proactively reduce inventory in Cisco's supply base.

If the players in the Beer Game were able to use sentiment analysis, it would be clear that there is a one-time shift in sentiment, which could be rapidly detected along the supply chain and responded to. Over time, sentiment settles, indicating that a return to a stable level of production is required, although at a different level than before. Used in this way, sentiment analysis, 
coupled with forecasting and an understanding of the supply and distribution chain, can be used to guide development of appropriate levels of manufacturing and inventory holding, without the need for overt coordination or collaboration over the chain.

A manufacturer is also able to evaluate consumer sentiment for particular features or models, allowing them to advance development of specific components or features into products, before such information flows through the supply chain. This may enable the manufacturer to compete with other firms, by releasing new products or models to consumers rapidly before competitors are able to manufacture competing products, or by dis-intermediating the supply chain and supplying directly to the consumer or through new sales channels, competing with their traditional channel partners.

Where a product is not available at the retail phase of the supply chain there is no apparent demand at the retailer (i.e., the retailer is not making sales as they do not have inventory, therefore it is impossible to measure demand based on sales). Sentiment analysis is still able to determine whether or not there is actual market-based demand for that product, allowing the supply base to react and accommodate this demand.

In this way, sentiment analysis is a method for gaining greater visibility of market demand even for upstream firms that are distant from consumer demand, allowing firms to proactively prepare for changes in dependent demand as it propagates down the supply chain in response to independent demand changes in the marketplace. This method represents a different paradigm to VMI, CPFR, or POS information sharing, all of which rely on collaboration and reliance on partners. Rather than rely on other firms (where many factors are outside of their control), a single firm can act autonomously to build a sentiment-analysis-based system embedded into their existing $\mathrm{S} \& O P$ process to improve firm-level responses to market changes with minimal disruption to existing operations, allowing them to proactively prepare for changes in orders along the supply chain, before they happen, based on detected changes in consumer demand.

\section{FUTURE RESEARCH DIRECTIONS}

Sentiment analysis is a relatively new field and only applied in practical scenarios in recent years. The need to process and understand natural language to detect polarity about a product is a complex task not yet adequately solved to guarantee an accurate result. Discipline specific applications (e.g., movies) demonstrate the success and benefit of sentiment analysis, whereas the market analysis for a supply chain with all its parts inhabits a higher complexity with the need formore advanced algorithms. As shown above, the ambiguity and context-specific meaning of terms can influence the outcome of the sentiment analysis; especially if stylistic features like irony or sarcasm are used. The phrase "go read the book" may be very positive in a book review, but turns out to be negative if it is said about a movie (Pang \& Lee, 2008).

The relevance of information is not equal for everyone in the supply chain; especially if parts are also used in other products that contribute to other supply chains. Suppliers can learn from feature descriptions, e.g., "the quality of a lens that is used in multiple products and brands." Sentiment analysis may also prove useful in uncovering links between markets over different product lines. If a component is used in both a low-value product and a high-value product, and the component is poorly received (i.e., one that consumers are critical of), the impact on sales may be greater in the high-value product. Thus, the analysis may prove more useful for suppliers upstream than for retailers.

To gain marketplace insight and improve supply chain planning, sentiment analysis is only one tool among many and should not be considered as a 'silver bullet' but should instead be integrated into S\&OP processes within the firm. This is an interesting area for further research and should prove to be a valuable contribution to practice and 
theory. First, the increase of user posting reviews and comments may cause many non-objective opinions that should not be considered. Second, the impact factor of reviews and comments is crucial in deriving relevant information as feedback.

Finally, the results from sentiment analysis must be aligned with traditional business intelligence methods; e.g., data mining methods. The current state-of-the-art of sentiment analysis with its high error of misinterpreting the emotion in the text can still be useful to enhance the forecast and to be used as a trigger for events and movements; however, supervision by experienced sales people and market analysts remains necessary. Work to integrate social media, including applications of sentiment analysis, in supply chains just recently started and will be one of the next relevant topics with respect to support decision-making (Mayeh et al., 2012; inter alia).

\section{CONCLUSION}

We have outlined a framework that demonstrates how firms without direct consumer contact can perceive markets events and occurrences and evaluate the overall sentiment, and draw inferences valuable in their supply management (Zitnik, 2012). We present sentiment analysis as a future feasible, low-cost choice for firms to develop their business intelligence to support supply chain management. The use of sentiment analysis allows firms to change their supply management structures dynamically in preparation for market changes which would otherwise take time to propagate along the supply chain. Such changes provide resilience to market-driven changes without the need to collaborate or coordinate with business partners in the supply chain, an increasingly important ability as modern supply chains becoming increasingly fractured due to outsourcing and globalisation. The framework was outlined with reference made to the Beer Game, to contrast sentiment analysis to existing methods for supplier firms to gain market information.

\section{REFERENCES}

Abbasi, M.-A., Chai, S.-K., Liu, H., \& Sagoo, K. (2012). Real-world behavior analysis through a social media lens. In S. J. Yang, A. M. Greenberg, \& M.Endsley (Eds.), Social Computing, Behavioral - Cultural Modeling and Prediction (pp. 18-26). Berlin: Springer. Retrieved from http://link.springer.com/chapter/10.1007/978-3-642-29047-3_3

Allen, J. F. (1995). Natural language understanding. Redwood City, CA: Benjamin/Cummings.

Baccianella, S., Esuli, A., \& Sebastiani, F. (2010). Sentiwordnet 3.0: An enhanced lexical resource for sentiment analysis and opinion mining. In Proceedings of the Seventh Conference on International Language Resources and Evaluation (LREC'10) (pp. 2200-2204). Valletta, Malta: European Language Resources Association(ELRA).

Boese, S., Reiners, T., \& Wood, L. C. (in press). Semantic document networks to support concept retrieval. In J. Wang (Ed.), Encyclopedia of Business Analytics and Optimization. Hershey, PA: IGI Global.

Esper, T. L., Ellinger, A. E., Stank, T. P., Flint, D. J., \& Moon, M. (2010). Demand and supply integration: A conceptual framework of value creation through knowledge management. Journal of the Academy of Marketing Science, 38(1), 5-18. doi:10.1007/s11747-009-0135-3

Helms, M. M., Ettkin,L. P., \& Chapman, S. (2000). Supply chain forecasting - Collaborative forecasting supports supply chain management. Business Process Management Journal, 6(5), 392-407. doi:10.1108/14637150010352408

Koenig, J.-P., Mauner, G., Bienvenue, B., \& Conklin, K. (2008). What with? The anatomy of a (proto)-role. Journal of Semantics, 25(2), 175-220. doi:10.1093/jos/ffm013 
Kumar, A., \& Sebastian, T. M. (2012). Sentiment analysis: A perspective on its past, present and future. [IJISA]. International Journal of Intelligent Systems and Applications, 4(10), 1. doi:10.5815/ ijisa.2012.10.01

Landauer, T. K. (2007). Handbook of latent semantic analysis. Lawrence Erlbaum Associates.

Lee, H. L. (2004). The Triple-A supply chain. Harvard Business Review, 82(10), 102-112. PMID:15559579

Lee, H. L., Padmanabhan, V., \& Whang, S. (1997). The bullwhip effect in supply chains. Sloan Management Review, 38(3), 93-102.

Liu, Y., Jin, J., Ji, P., Harding, J. A., \& Fung, R. Y. K. (2013). Identifying helpful online reviews: A product designer's perspective. Computer Aided Design, 45(2), 180-194. doi:10.1016/j. cad.2012.07.008

Madison, M., Cowell, A., Butner, R. S., Fligg, K., Piatt, A., McGrath, L., \& Ellis, P. (2012). Knowledge encapsulation framework for technosocial predictive modeling. Security Informatics, 1(1), 10. doi:10.1186/2190-8532-1-10

Mayeh, M., Scheepers, R., \& Valos, M. (2012). Understanding the role of social media monitoring in generating external intelligence. ACIS 2012 : Location, location, location : Proceedings of the 23rd Australasian Conference on Information Systems 2012, 1-10.

Montoyo, A., Martínez-Barco, P., \& Balahur, A. (2012). Subjectivity and sentiment analysis: An overview of the current state of the area and envisaged developments. Decision Support Systems, 53(4), 675-679. doi:10.1016/j.dss.2012.05.022

Nadkarni, P. M., Ohno-Machado,L., \& Chapman, W. W. (2011). Natural language processing: An introduction. Journal of the American Medical Informatics Association, 18(5), 544-551. doi:10.1136/ amiajnl-2011-000464 PMID:21846786
O'Leary, D. E. (2011). Blog mining-review and extensions: "From each according to his opinion. Decision Support Systems, 51(4), 821-830. doi:10.1016/j.dss.2011.01.016

Pang, B., \& Lee, L. (2008). Opinion mining and sentiment analysis. Foundations and Trends in Information Retrieval, 2(1-2), 1-135. doi:10.1561/1500000011

Prabowo, R., \& Thelwall, M. (2009). Sentiment analysis: A combined approach. Journal of Informetrics, 3(2), 143-157. doi:10.1016/j. joi.2009.01.003

Qin, L. (2011). Word-of-blog for movies: A predictor and an outcome of box office revenue? Journal of Electronic Commerce Research, 12(3), 187-198.

Sterman, J. D. (1989). Modeling managerial behavior: Misperceptions of feedback in a dynamic decision making experiment. Management Science,35(3),321-339. doi:10.1287/mnsc.35.3.321

Strapparava, C., \& Valitutti, A. (2004). WordNetAffect: An affective extension of WordNet. In Proceedings of the 4th International Conference on Language Resources and Evaluation (LREC 2004) (pp. 1083-1086). Presented at the 4th International Conference on Language Resources and Evaluation (LREC 2004), Lisbon, Portugal.

Taboada, M., Brooke, J., Tofiloski, M., Voll, K., \& Stede, M. (2011). Lexicon-Based Methods for Sentiment Analysis. Computational Linguistics, 37(2), 267-307. doi:10.1162/COLI_a_00049

Tjong Kim Sang, E. F., \& De Meulder, F. (2003). Introduction to the CoNLL-2003 Shared Task: Language-Independent Named Entity Recognition. In M. Daelemans, Walter; Osborne (Ed.), Proceedings of the conference on Natural language learning at HLT-NAACL 2003 (pp. 142-147). Morristown, NJ: Association for Computational Linguistics. 
Tsytsarau, M., \& Palpanas, T. (2012). Survey on mining subjective data on the Web. Data Mining and Knowledge Discovery, 24(3), 478-514. doi:10.1007/s10618-011-0238-6

Wille, R. (2009). Restructuring lattice theory: An approach based on hierarchies of concepts. In S. Ferré, \& S. Rudolph (Eds.), Formal Concept Analysis (Vol. 5548, pp. 314-339). Berlin: Springer. doi:10.1007/978-3-642-01815-2_23

Yu, S., \& Kak, S. (2012). A survey of prediction using social media. arXiv:1203.1647. Retrieved from http://arxiv.org/abs/1203.1647

Zeng, L., Li, L., \& Duan, L. (2012). Business intelligence in enterprise computing environment. Information Technology and Management, 13(4), 297-310. doi:http://dx.doi.org.dbgw.lis.curtin. edu.au/10.1007/s10799-012-0123-z

Zitnik, M. (2012). Using sentiment analysis to improve business operations. XRDS: Crossroads, 18(4), 42-43. doi:10.1145/2173637.2173655

\section{ADDITIONAL READING}

Egozi, O., Markovitch, S., \& Gabrilovich, E. (2011). Concept-based information retrieval using explicit semantic analysis. ACM Transactions on Information Systems, 29(2), 1-8.

Greenberg, P. (2010). The impact of CRM 2.0 on customer insight. Journal of Business \& Industrial Marketing, 25(6), 410-419. doi:10.1108/08858621011066008

Song, M., \& Wu, Y.-F.B. (2009). Research on text and Web mining technologies. Hershey: IGI Global.

Sugumaran, V. (2011). Applied semantic Web technologies. CRC Press.
Wilson, T., Wiebe, J., \& Hoffmann, P. (2009) Recognizing contextual polarity: An exploration of features for phrase-level sentiment analysis. Computational linguistics, 35(3), 399-433.

Wriedt, S., Reiners, T., \& Ebeling, M. (2008). How to teach and demonstrate topics of supply chain management in virtual worlds. In Proceedings of ED-MEDIA 2008. World Conference on Educational Multimedia, Hypermedia \& Telecommunications, Vienna, Austria (pp. 5501-5508). Chesapeake, VA: AACE.

\section{KEY TERMS AND DEFINITIONS}

Beer: A beverage that is fun to drink and enhances knowledge creation activities in universities around the globe.

Bullwhip Effect: An observed trend of increasingly larger oscillations in inventory the further a firm is from consumers, in response to changes in consumer demand.

Concept: One or multiple words associated with a category that was generated by the abstraction of common characteristics from a range of particular ideas, while removing the uncommon characteristics. The remaining common characteristic is similar to all of the different individuals and represents the meanings of the ideas.

Concept Retrieval: The ability to query a document and extract particular segments of text that match concepts or ideas provided by a user.

Semantic Analysis: The elicitation of knowledge from documents, accounting for the context and understanding. The units that are extracted are arranged and grouped within meaningful categories.

Sentiment Analysis: Application of natural language processing, computational linguistics, and text analytics to identify and extract subjective information in text materials. 
Sentiment Classification: Classifying the polarity of a given text in the document, sentence, or feature/aspect level; e.g., emotional states.

Supply Chain: Sequence of activities conducted in separate companies transporting a materials flow to consumers while progressively transforming it into the desired products. Information from the market flows to the producers.
Text Analysis: The process of deriving meaningful information from the data and ideas expressed within the document. It includes metainformation, structural information, and content information. 